Published in Mathematical Biosciences 191 (2004) 207-225

\title{
Mathematical analysis of competition between sensory ganglion cells for neurotrophic factor in the skin
}

\author{
R. Kohli a, J.-P. Gabriel ${ }^{\text {b }}$, P.G.H. Clarke ${ }^{\text {a,* }}$ \\ a Département de Biologie cellulaire et de Morphologie, Université de Lausanne, Rue du Bugnon 9 , \\ CH-1005 Lausanne, Switzerland \\ ${ }^{\mathrm{b}}$ Département de Mathématiques, Université de Fribourg, CH-1700 Fribourg, Switzerland
}

Received 3 April 2003; received in revised form 10 May 2004; accepted 30 June 2004

\begin{abstract}
A model is presented of competition between sensory axons for trophic molecules (e.g. a neurotrophin such as NGF), produced in a region of skin small enough to permit their free diffusion throughout it; e.g., a touch dome, or a vibrissal follicle hair sinus. The variables specified are the number of high affinity trophic factor receptors per axon terminal and the concentration of trophic factor in the extracellular space. Previous models of this class predicted the loss of all the axons innervating the region except the one requiring least trophic factor for its maintenance, even with high rates of trophic factor production. In the present model, we have imposed upper limits to axonal growth, thereby introducing new equilibria, and we show by a global analysis using LaSalle's theorem, and also by local analysis, that several axons can then coexist if the rate of production of trophic molecules is sufficiently high.
\end{abstract}

(c) 2004 Elsevier Inc. All rights reserved.

Keywords: Axon; Coexistence; Competition; Competitive exclusion; Modeling; Neurotrophin; Skin; Trophic factor

\footnotetext{
* Corresponding author. Tel.: +41 21692 5120; fax: +41 216925105.

E-mail address: peter.clarke@ibcm.unil.ch (P.G.H. Clarke).
} 


\section{Introduction}

Throughout the nervous system, competition between developing axons is a major determinant of how they share their target territory between them. It strongly influences which of their parent cell bodies will survive the acute phase of massive cell death that eliminates $30-70 \%$ of the neurons in most neuronal populations while connections are being formed [1], and even after the period of neuronal death axons continue to compete. Even in adults, section of a sensory nerve is followed by the ingrowth of axons from adjacent nerves into the denervated territory, presumably due to the elimination of some kind of competitive or repulsive influence that had been exerted by the nerve before it was sectioned. Both during and after the neuronal death, these interaxonal interactions are believed to be due largely to competition for 'neurotrophic' (or simply 'trophic') molecules produced in the axonal target territory. This paper is concerned with the competition between sensory axons during both periods - during and after neuronal death.

\subsection{Interaxonal competition as a determinant of neuronal death in sensory ganglia}

The classic interpretation, which we shall call the competitive matching hypothesis, is that neuronal death is regulated by competition between neurons for a trophic factor produced in their axonal target territory and that it serves to match the number of neurons to the 'size' (or trophic factor producing capacity) of the target [1,2]. Whilst this view is too simple to account for the complexity of events in the central nervous system, where electrical activity and the receipt of afferents strongly influence neuronal survival [3-5], it is widely accepted for sensory ganglia for the following reasons. First, although axial level differences in size of spinal ganglia are not purely the result of neuronal death [8], there is less of it at the brachial and lumbar levels (which innervate the limbs) [9]. Second, enlarging the periphery by means of transplantation reduces neuronal death in sensory ganglia [2,10-12]. Third, when a few ganglia are ablated or are prevented from developing by partial lesions of the neural crest, the adjacent ganglia exhibit hypertrophy and increased neuronal numbers, presumably because of reduced competition between the ganglia $[13,14]$. Fourth, even the developing sensory neurons that normally survive die if deprived of an essential trophic factor during the cell death period. For many sensory neurons including those expressing substance-P the trophic factor is NGF, but some classes of sensory neurons depend on other members of the NGF family (called 'neurotrophins'), notably BDNF and NT-3 [15-20], or members of other protein families [21]. Fifth, NGF-dependent sensory neurons obtain their NGF exclusively from their target territory, where it is synthesized, and remove it by retrograde transport in sufficiently large quantities to greatly deplete its concentration in the target [22,23]. This provides a possible basis for competition, as is illustrated by the fact that removing the sympathetic innervation of a region can lead to an almost threefold increase in the level of NGF in the sensory ganglia innervating it [24]. Sixth, raising the concentration of NGF (or other neurotrophins) in the axonal target region above normal levels reduces neuronal death, causing ganglionic hypertrophy [6,7]. Most such experiments involved the systemic administration of exogenous trophic factor, but Albers et al. [25,26] showed that transgenic mice overexpressing NGF in the epidermis (but not elsewhere) were subject to greatly reduced death of sensory ganglion neurons, and a similar approach has subsequently been used to show reduced ganglion neuron death following cutaneous overexpression of NT-3 [19] or BDNF [18]. 


\subsection{Competition between sensory axons without neuronal death}

When the neuronal death period has ended, the sensory axons continue to compete for trophic molecules. This phase of competition has been studied less extensively than the neuronal death phase, but the occurrence of some kind of competition, even in adults, is implied by the classical phenomenon of collateral sprouting, in which denervation of a skin territory by section of a sensory nerve leads to the outgrowth of new axonal branches (mainly from C-fibers) from adjacent nerves into the denervated territory [27,28]. Moreover, NGF continues to be expressed in the skin of adult rodents [29], depletion or inactivation of NGF prevents the collateral sprouting phenomenon, and intradermal injections of purified NGF increased the sprouting and were even able to evoke sprouting de novo in normally innervated skin $[28,30]$.

\section{Choice of system to model and approach}

Interaxonal competition is believed to occur throughout the nervous system, but we choose to focus on the competition between sensory axons for peripheral neurotrophins because it is particularly well established, and because it is free from many of the complications that make competition in other systems difficult to analyse. Since sensory neurons do not receive synapses, their survival is not influenced by the complicating factor of afferent control. In the case of motoneurons, there is evidence against competition (at least, in the straightforward sense), because in Xenopus frogs reared with a single hindlimb innervated by both sides of the spinal cord, an almost normal number of motoneurons can survive on both sides [31]. However, in the very same experimental animals, the total number of sensory neurons surviving on both sides approximates the number on one side in normal frogs [32], as the competition hypothesis would predict. Another complication in the case of virtually all neurons except sensory neurons is that their axonal competition is strongly influenced by electrical activity, possibly involving activity-mediated modulation of the synthesis and release by the target cells of several different trophic factors [33]. In contrast, NGF-production in the skin is independent of the innervating axons and of their electrical activity $[34,35]$.

\subsection{Choice to model competition for a single trophic factor in a small region of skin}

At least five different trophic factors - the neurotrophins NGF, BDNF and NT-3 and NT-4; and glial cell line-derived neurotrophic factor (GDNF) - are expressed in the skin and can mediate the survival of sensory neurons [21,23,36,37]. Each of these trophic factors normally occurs in the extracellular space as a homodimer, and binds to low and high affinity receptors [38]. In the case of the neurotrophins, each binds to the same low affinity receptor (whose identity as the p75 neurotrophin receptor is currently controversial-[39]), but almost specifically to a single high affinity receptor that is a member of the trk family (NGF to trkA (or trk), BDNF to trkB, NT-3 to trkC although also to trkA and trkB with lower affinity, NT-4 to trkB) [40]. At any given moment most sensory neurons depend on only a single trophic factor, and while some neurons switch their dependency, for example from BDNF or NT-3 to NGF [36] or from NGF to GDNF [41], at least $40 \%$ depend exclusively on NGF [42]. Mouse strains lacking a particular trophic 
factor or its corresponding high affinity receptor lose the corresponding population of sensory neurons [42].

We therefore propose a model involving competition between sensory axons for a single trophic factor. It could in principle be applied to any of the four neurotrophins mentioned here, or to GDNF, but to simplify discussion we shall assume it to be a neurotrophin, and in some cases we shall focus specifically on NGF. Since it is not our present purpose to model the spatial aspects of axonal competition, we envisage the competition as occurring within a region of skin sufficiently small to permit free diffusion of neurotrophin throughout it, leading to a relatively uniform spatial distribution of the trophic molecules. Such a region could be a touch-dome (also called 'haarscheibe') or a vibrissal follicle hair sinus. Touch domes are heavily innervated sensory receptor structures, about $50 \mu \mathrm{m}$ across, and innervated generally by a small number of axons, e.g. 1-3 in the cheek pouch mucosa of young adult hamsters [43]. The axons terminate on Merkel cells. Touch domes are difficult to identify during the first days of interaxonal competition, which occurs throughout the period of naturally occurring neuronal death (last week of embryonic life in mouse trigeminal ganglion- [44] and continues, without further neuronal death, into the first few weeks of the postnatal period.

Touch domes become clearly recognizable (in skin from the flank and back of mice) at about embryonic day 17 [45]. In transgenic mice overexpressing NT-3 in the skin, the innervation density of touch domes is greatly enhanced, apparently through reduced interaxonal competition for NT-3 [19].

Vibrissal follicle hair sinuses are spindle-shaped structures, about $500 \mu \mathrm{m}$ in diameter, containing a vibrissal follicle surrounded by a mesenchymal sheath and embedded in a vascular sinus. The follicles are heavily innervated and express NGF; and cutaneous overexpression of NGF increases their innervation density [46].

\subsection{Modeling axonal competition as a determinant of neuronal death}

The competition model developed here finds its roots in [66] and is a modification of the one given in [47]. While our basic model relates to competition between axons, it is directly relevant to neuronal death, and in particular to the competitive matching hypothesis, which has never been formalized in an explicit model. Any such model must incorporate the notion that the neurons that die are not inherently maladapted, but lose in competition between almost-equals. The question arises, then, as to how minor differences between axons could be used to select for the survival of just the right number of neurons for a given target. In one class of models, involving what we shall call one-stage competition, selection would occur directly, without any prior change in competitive capacity. For example, a region of skin with rate of neurotrophin production $Q$ might be innervated by sensory neurons each requiring for survival a rate $U$ of neurotrophin-uptake. Clearly, the maximum number that could survive would be $N=\frac{Q}{U}$, independently of the initial number. At first sight, this model may seem to provide an ideal mechanism for making neuron survival proportional to peripheral NT production, but there are at least three serious problems with it. First, there is abundant evidence that the rate of uptake is not constant, but varies with axonal growth or regression under the influence of trophic molecules. Second, their production would have to be preprogrammed with inordinate precision; the slightest deviation from the 'correct' level would greatly affect the number of surviving neurons. Third, the dynamics of the model 
are problematic. Although $N=\frac{Q}{U}$ sets an obvious maximum to the number of neurons that could survive, it is by no means clear that this would be the actual number. When trophic factor is removed completely from neurons in culture, there is a time delay of more than $12 \mathrm{~h}$ before neuronal death occurs. During such a delay in vivo, excessive competition would bring the uptake of most or all neurons below the critical level, causing the final number to be much lower than $N=\frac{Q}{U}$.

Because of these problems, we postulate a model of neuronal death due to two-stage competition. The idea is that an initial stage of competition between axons would affect not cell death but the capacity to take up trophic factor, leading ultimately to major differences between the uptake rates of different axons, even if the rates were initially similar. In the second stage, neurons would indeed die, not because their uptake was slightly lower than that of the survivors, but because their axonal arbors had so diminished that they were receiving almost no trophic factor at all.

Our previous model of interaxonal competition, which incorporates these features, solves the above problems [47]. But it introduces a new one: when competition modifies the competitive capacity, the fittest axon becomes so strong that it eliminates all the others. This competitive exclusion appears indeed to occur in certain situations, such as the elimination of polyneuronal innervation of muscle fibers during development $[49,50]$ or the mosaic organization of skin innervation by rapidly adapting terminals in salamanders [51], but not in others such as the overlapping arrangement of axonal fields in the skin of frogs [52]. Moreover, this earlier model predicted that competitive exclusion would still occur even with very high levels of trophic factor production, implying that the total number of axons innervating a region would not be increased, but experiments involving the overexpression of neurotrophins in the skin have shown that innervation density does increase in the skin in general [46], and in touch domes in particular [19].

We here avoid competitive exclusion by setting a limit to the growth of each axon, which profoundly complicates the behavior of the model, introducing new equilibria corresponding to the persistence of multiple innervation when the production of trophic factor is sufficiently high. We adopt a mathematical rather than a computational approach, partly because there is currently insufficient data for us to be able to specify the precise parameters and functions that would be needed for computer simulations. The generality of our mathematical analysis avoids this difficulty. The conclusions of this paper were briefly reported in a conference proceedings [53].

\section{Development of a mathematical model}

\subsection{The role of neurotrophin receptors}

Neurotrophins are not merely survival factors, but have immediate positive effects on the growth and maintenance of axon terminals [54-56]. For simplicity, we here focus on NGF, but the same principles apply to the other neurotrophins.

Receptor binding studies have indicated the equilibrium dissociation constants of the specific high affinity NGF receptor $\left(K_{d \mathrm{H}} \approx 10^{-11} \mathrm{M}\right)$ and of the low affinity receptor $\left(K_{d \mathrm{~L}} \approx 10^{-9} \mathrm{M}\right)$ [57,40]. Cultured chick spinal ganglion cells have 1000-3000 high affinity NGF receptors per cell and 23000-45000 low affinity receptors [58]. The binding of NGF to the high affinity receptor dimerizes trkA and activates the tyrosine kinase activity of each trkA, leading to a chain of second 
messenger reactions in the terminal. Binding to the low affinity receptor can also initiate second messenger reactions, and in many cases these promote neuronal death rather than preventing it [38], but in the skin during the phase of competition between sensory axons the NGF concentrations appear to be only of the order of $10^{-12} \mathrm{M}$ [23], which is probably too low to significantly affect signaling from the low affinity receptor (but see Section 5). We shall therefore assume that the growth promoting effect of NGF is a function of the proportion of bound high affinity receptors.

\subsection{Equations for binding of $N G F$}

We thus consider the competition between $\mathrm{n}$ axons (or axonal branches, or terminals) for NGF in a touch dome (or similar small region) where the concentration $S(t)$ of NGF in the extracellular space is sufficiently uniform for us to be able to ignore any spatial variation. Then, the proportions of bound high and low affinity receptors, respectively $P_{\mathrm{H}}(S)$ and $P_{\mathrm{L}}(S)$, can conventionally be expressed by the Michaelis-Menten (or Langmuir) equation as $P_{\mathrm{H}}(S)=\frac{S}{K_{d \mathrm{H}}+S}$ and $P_{\mathrm{L}}(S)=\frac{S}{K_{d \mathrm{~L}}+S}, K_{d \mathrm{H}}$ and $K_{d \mathrm{~L}}$ being the equilibrium dissociation constants of the high and low affinity receptors [59]. This assumes the internalization of NGF by endocytosis to be negligible in comparison to its constant exchange between the bound compartment and the extracellular space, which is probably true for NGF $[58,60]$. But even if internalization were rapid, we can show that the form of the equation would not change. In unit time, $S K_{1}(1-P(S))$ molecules will be bound, $K_{-1} P(S)$ molecules will dissociate, and $h P(S)$ will be internalized, where $K_{1}$ and $K_{-1}$ are the rate constants of association and dissociation and $h$ is also a constant. Hence, $\left(K_{-1}+h\right) P(S)=S K_{1}(1-P(S))$ and $P(S)=\frac{S}{S+\frac{K_{-1}+h}{K_{1}}}$. Thus $K_{d}=\frac{K_{-1}}{K_{1}}$ would merely have to be replaced by $\frac{K_{-1}+h}{K_{1}}$. Despite the plausibility of the Michaelis-Menten equation, we shall not need to assume its exact validity, but shall make the weaker assumption that $P_{\mathrm{H}}(S)$ and $P_{\mathrm{L}}(S)$ are increasing functions of $S$. Also, it follows from the definitions of $P_{\mathrm{H}}(S)$ and $P_{\mathrm{L}}(S)$ that $P_{\mathrm{H}}(0)=P_{\mathrm{L}}(0)=0$ and $P_{\mathrm{H}}(\infty)=P_{\mathrm{L}}(\infty)=1$.

\subsection{Equation for axonal growth or regression}

Of the many axonal parameters that grow or decay, we choose to deal with the number of high affinity receptors, since it is these that mediate axonal growth and NGF-removal. Let $x_{i}(t)$ be the number of these receptors on axon $i$. We assume that the rate of growth will depend on the level of some second messenger in the receptor-bearing (terminal) region of the axon, and that this will be an increasing function of the proportion $P_{\mathrm{H}}(S)$ of bound high affinity receptors. Since $P_{\mathrm{H}}(S)$ is likewise an increasing function of $S$, the level of second messenger $F=F(S)$ is one also. But since growth is negative in the presence of a low or zero level of NGF, we assume that the rate of growth will be proportional to $F(S)-F\left(S_{i}^{T}\right), S_{i}^{T}$ being the concentration of NGF required for zero growth of axon $i$. We assume that $S_{i}^{T}$ is a constant. Clearly $\frac{\mathrm{d} x_{i}(t)}{\mathrm{d} t}$ will also depend on $x_{i}(t)$. We therefore write

$$
\frac{\mathrm{d} x_{i}(t)}{\mathrm{d} t}=H_{i}\left(x_{i}(t)\right)\left[F(S(t))-F\left(S_{i}^{T}\right)\right] .
$$


We assume also that the growth rate will be zero when $x_{i}=0$ or when $x_{i}$ becomes so large that the capacity of the cell body and axon to maintain it becomes saturated. To express these conditions, we can write $H_{i}\left(x_{i}\right)=x_{i}\left(x_{i}-X_{i}\right) G_{i}\left(x_{i}\right), X_{i}$ being a constant and $G_{i}\left(x_{i}\right)$ any bounded, nonzero function of $x_{i}$. Hence,

$$
\frac{\mathrm{d} x_{i}(t)}{\mathrm{d} t}=x_{i}(t)\left(x_{i}(t)-X_{i}\right) G_{i}\left(x_{i}(t)\right)\left[F(S(t))-F\left(S_{i}^{T}\right)\right] .
$$

\subsection{Equation for the changing concentration of NGF}

Since the cutaneous production of NGF is not modulated by the innervating axons [34,35], and since the cutaneous cells express few if any high or low affinity NGF receptors [23], we shall assume that the rate of NGF production Q is constant, and that the only means of NGF elimination are by the innervating axons and by passive processes such as diffusion and degradation. NGF removal by axons involves receptor-mediated endocytosis. The importance in this of the low affinity receptors is uncertain, so we cannot ignore it. We assume that their number is maintained in constant proportion to that of the high affinity receptors, and that the endocytosis mediated by each kind is proportional to the number of bound receptors. Hence, the axonal removal rate $R_{\mathrm{a}}(S)=x_{i}\left[K_{\mathrm{H}} P_{\mathrm{H}}(S)+K_{\mathrm{L}} P_{\mathrm{L}}(S)\right]$. We express the passive removal rate as $R_{\mathrm{p}}(S)=A S$. Hence,

$$
\begin{aligned}
\frac{\mathrm{d} S(t)}{\mathrm{d} t} & =Q-R_{\mathrm{p}}(S(t))-R_{\mathrm{a}}(S(t))=Q-A S(t)-\left[K_{\mathrm{H}} P_{\mathrm{H}}(S(t))+K_{\mathrm{L}} P_{\mathrm{L}}(S(t))\right] \sum_{i=1}^{n} x_{i}(t) \\
& =Q-A S(t)-W(S(t)) \sum_{i=1}^{n} x_{i}(t),
\end{aligned}
$$

where $W(S)=K_{\mathrm{H}} P_{\mathrm{H}}(S)+K_{\mathrm{L}} P_{\mathrm{L}}(S)$.

\section{Mathematical analysis}

The preceding considerations lead to the following system:

$$
\left\{\begin{array}{l}
\dot{S}(t)=Q-A S(t)-W(S(t)) \sum_{i=1}^{n} x_{i}(t), \\
\dot{x}_{i}(t)=x_{i}(t)\left(X_{i}-x_{i}(t)\right) G_{i}\left(x_{i}(t)\right)\left(F(S(t))-F\left(S_{i}^{T}\right)\right), \quad i=1, \ldots, n,
\end{array}\right.
$$

where $W, F$ and $G_{i}, i=1, \ldots, n$ are real-valued and continuously differentiable functions defined over $\mathbb{R}_{+}=[0, \infty]$. Moreover, $W$ and $F$ are strictly increasing over $\mathbb{R}_{+}$and there exist numbers $\bar{W}$ and $\underline{G}_{i}, \bar{G}_{i}, i=1, \ldots, n$, such that $0=W(0)<W(S) \leqslant \bar{W}, 0=F(0)<F(S) \leqslant 1,0<\underline{G}_{i} \leqslant$ $G_{i}\left(x_{i}\right) \leqslant \bar{G}_{i}<\infty, i=1, \ldots, n$.

For $i=1, \ldots, n$, the parameters $Q, A, X_{i}$, are strictly positive and $S_{i}^{T}$ are non-negative, ordered according to $0 \leqslant S_{1}^{T} \leqslant S_{2}^{T} \leqslant \cdots \leqslant S_{n}^{T}<\infty$.

We introduce the notations $F_{i}^{T}=F\left(S_{i}^{T}\right), \quad W_{i}^{T}=W\left(S_{i}^{T}\right) \quad$ and $\quad D=\left\{\left(S, x_{1}, \ldots, x_{n}\right) \in\right.$ $\left.\mathbb{R}^{n+1} \mid S \geqslant 0,0 \leqslant x_{i} \leqslant X_{i}, i=1, \ldots, \mathrm{n}\right\}$, the latter being called physiological domain for obvious reasons. 


\subsection{Existence and unicity}

Theorem 1. For every given initial condition $S(0) \geqslant 0,0 \leqslant x_{i}(0) \leqslant X_{i}, i=1, \ldots, n$, system (1) admits a unique solution $\left(S(t), x_{1}(t), \ldots, x_{n}(t)\right)$ defined over $[0, \infty)$, such that for all $t>0$, either $S(t)>0$ and $0<x_{i}(t)<X_{i}$, or $x_{i}(t) \equiv 0$, or $x_{i}(t) \equiv X_{i}$ according to whether $0<x_{i}(0)<X_{i}$, or $x_{i}(0)=0$, or $x_{i}(0)=X_{i}$.

Proof. We first discuss system (1) in the interior $D^{\circ}$ of $D$. Since its right-hand member is continuously differentiable over the open set $D^{\circ}$, the general theory of differential systems [61] (p. 73) and [62] (Corollary 3.2) provides, for every initial condition in $D^{\circ}$, the existence and unicity of a solution (denoted $y(t)$ ) defined over a maximal right interval $J$. Moreover, the right-hand member being continuous over the closed set $D$, either $J=[0, \infty)$, or $J=[0, \delta]$ with $\delta>\infty$ and $y(\delta) \in \partial D$, or $J=[0, \delta)$ with $\delta<\infty$ and $\|y(t)\| \rightarrow \infty$ as $t \rightarrow \delta$. As long as $t \in J$, the following integral representations hold:

$$
\begin{aligned}
x_{i}(t) & =x_{i}(0) \exp \left[\int_{0}^{t}\left(X_{i}-x_{i}(u)\right) G_{i}\left(x_{i}(u)\right)\left(F(S(u))-F_{i}^{T}\right) \mathrm{d} u\right] \\
& =X_{i}-\left(X_{i}-x_{i}(0)\right) \exp \left[-\int_{0}^{t} x_{i}(u) G_{i}\left(x_{i}(u)\right)\left(F(S(u))-F_{i}^{T}\right) \mathrm{d} u\right] .
\end{aligned}
$$

The second representation of $x_{i}(t)$ follows from $\dot{x}_{i}(t)=\frac{\mathrm{d}}{\mathrm{d} t}\left(X_{i}-x_{i}(t)\right)$ and the differential equation for $\dot{x}_{i}(t)$ rewritten for the unknown function $\left(X_{i}-x_{i}(t)\right)$. Clearly, for $t \in J$, we get $0<x_{i}(t)<X_{i}$. Moreover, $S(t)$ remains strictly positive over $J$; indeed, let us denote by $t^{*}$ the first non-negative time for which $S\left(t^{*}\right)=0$. Since $S(0)>0, \dot{S}\left(t^{*}\right) \leqslant 0$ but the equation for $S$ entails $\dot{S}\left(t^{*}\right)=Q>0$, a contradiction. Consequently, for all $t \in J, y(t) \in D^{\circ}$, ruling out the configuration $J=[0, \delta]$ with $\delta<\infty$. Since $x_{i}(t), i=1, \ldots, n$, are bounded over $J$, the only way to achieve $J=[0, \delta)$ with $\delta<\infty$ is $\varlimsup_{t \rightarrow \delta} S(t)=\infty$. Thus one can find a sequence $0<u_{k} \uparrow \delta$ such that $S\left(u_{k}\right)$ is strictly increasing and $S\left(u_{k}\right) \rightarrow \infty$ for $k \rightarrow \infty$. For each $k$, the mean value theorem provides $u_{k}<v_{k}<u_{k+1}$ for which $\dot{S}\left(v_{k}\right)>0$. However, in

$$
\dot{S}\left(v_{k}\right)=Q-A S\left(v_{k}\right)-W\left(S\left(v_{k}\right)\right) \sum_{i=1}^{n} x_{i}\left(v_{k}\right),
$$

the first term is constant, the third one is negative and the second one goes to $-\infty$, a contradiction. Therefore we can conclude that $J=[0, \infty)$.

For initial values on the boundary of $D$, one can extend (even linearly) the right-hand member of system (1) to a continuously differentiable function defined on an open set $D^{*}$ containing $D$. Each time $x_{i}(0)$ is 0 or $X_{i}$, the preceding integral representations entail that the unique solution is $x_{i}(t)=x_{i}(0)$ over the maximal right interval of existence. Moreover, if $S(0)=0$, then $\dot{S}(0)=Q>0$ and $S(t)$ is thus strictly positive over some interval of the form $(0, \alpha)$. It is now clear that the same argumentation as before can be repeated by starting with some $t_{0}>0$ and reducing the dimension each time an $x_{i}$ is constant.

Consequently, every solution of system (1) starting in $D$ at $t=0$ is uniquely defined for all $t \geqslant 0$ and remains in $D$. 


\subsection{Critical points and local stability}

Straightforward considerations show that the set $E$ of critical points of system (1) in $D$ is given by $E_{1} \cup E_{2}$ where:

$$
\begin{aligned}
E_{1}= & \left\{\left(S^{0}, x_{1}^{0}, \ldots, x_{n}^{0}\right) \in D \mid x_{i}^{0}=0 \text { or } x_{i}^{0}=X_{i}, \quad i=1, \ldots, n \text { and } S^{0}\right. \text { with } \\
& \left.Q-A S^{0}=W\left(S^{0}\right) \sum_{i=1}^{n} x_{i}^{0}\right\} \text { and } \\
E_{2}= & \cup_{r=1}^{n}\left\{\left(S^{0}, x_{1}^{0}, \ldots, x_{n}^{0}\right) \in D \mid S^{0}=S_{r}^{T}, \quad x_{i}^{0}=0 \quad \text { or } \quad x_{i}^{0}=X_{i},\right. \\
& \left.i=1, \ldots, n, \quad i \neq r \text { and } x_{r}^{0}=\frac{Q-A S^{0}}{W\left(S^{0}\right)}-\sum_{i=1, i \neq r}^{n} x_{i}^{0}\right\} .
\end{aligned}
$$

The set $E_{1}$ contains exactly $2^{n}$ elements. For each $1 \leqslant r \leqslant n$, the corresponding subset of $E_{2}$ contains $2^{n-1}$ points but not necessarily in $D$ since $x_{0}^{r}$ may not belong to $\left[0, X_{r}\right]$. Consequently $E$ contains at most $(n+2) 2^{n-1}$ elements.

Our smoothness assumptions about the right-hand member of system (1) imply that local asymptotic stability and instability of critical points can be deduced from the eigenvalues of the Jacobian matrix at the point of interest [63].

Theorem 2. Let $\left(S^{0}, x_{1}^{0}, \ldots, x_{n}^{0}\right)$ be a critical point of system (1) in the physiological domain, $J^{0}=J\left(S^{0}, x_{1}^{0}, \ldots, x_{n}^{0}\right)$ its Jacobian matrix at $\left(S^{0}, x_{1}^{0}, \ldots, x_{n}^{0}\right), \sigma\left(J^{0}\right)$ its spectrum and $\Lambda=$ $\max _{i}=0, \ldots, n\left\{\operatorname{Re}\left(\lambda_{i}\right) \mid \lambda_{i} \in \sigma\left(J^{0}\right)\right\}$. Then $\Lambda<0$ if and only if $P_{1} \cup P_{2} \cup P_{3}=\{1, \ldots, n\}$, where:

$$
\begin{aligned}
& P_{1}=\left\{j \in\{1, \ldots, n\} \mid S_{j}^{T}>S^{0} \text { and } x_{j}^{0}=0\right\}, \\
& P_{2}=\left\{j \in\{1, \ldots, n\} \mid S_{j}^{T}<S^{0} \text { and } x_{j}^{0}=X_{j}\right\}, \\
& P_{3}=\left\{j \in\{1, \ldots, n\} \mid S_{j}^{T}=S^{0} \text { and } 0<x_{j}^{0}<X_{j}\right\} .
\end{aligned}
$$

Proof. The Jacobian matrix has the form:

$$
J^{0}=\left(\begin{array}{ccccc}
J_{00} & J_{01} & \ldots & \ldots & J_{0 n} \\
J_{10} & J_{11} & 0 & \ldots & 0 \\
\vdots & 0 & \ddots & \ddots & \vdots \\
\vdots & \vdots & \ddots & \ddots & 0 \\
J_{n 0} & 0 & \ldots & 0 & J_{n n}
\end{array}\right),
$$


where the elements $J_{i j}$ are given by:

$$
\begin{aligned}
J_{00} & =-A-\frac{\mathrm{d} W}{\mathrm{~d} S}\left(S^{0}\right) \sum_{i=1}^{n} x_{i}^{0}, \\
J_{0 j} & =-W\left(S^{0}\right), \quad j=1, \ldots, n, \\
J_{i 0} & =x_{i}^{0}\left(X_{i}-x_{i}^{0}\right) G_{i}\left(x_{i}^{0}\right) \frac{\mathrm{d} F}{\mathrm{~d} S}\left(S^{0}\right), \quad i=1, \ldots, n, \\
J_{i i} & =\left[\left(X_{i}-2 x_{i}^{0}\right) G_{i}\left(x_{i}^{0}\right)+x_{i}^{0}\left(X_{i}-x_{i}^{0}\right) \frac{\mathrm{d} G}{\mathrm{~d} x_{i}}\left(x_{i}^{0}\right)\right]\left(F\left(S^{0}\right)-F_{i}^{T}\right), \quad i=1, \ldots, n .
\end{aligned}
$$

For any critical point in $E_{1}$, we have:

$$
J_{i 0}=0, i=1, \ldots, n \quad \text { and } \quad J_{i i}=\left(X_{i}-2 x_{i}^{0}\right) G_{i}\left(x_{i}^{0}\right)\left(F\left(S^{0}\right)-F_{i}^{T}\right), \quad i=1, \ldots, n .
$$

$J^{0}$ is a triangular matrix whose eigenvalues are given by:

$$
\begin{aligned}
& \lambda_{0}=-A-\frac{\mathrm{d} W}{\mathrm{~d} S}\left(S^{0}\right) \sum_{i=1}^{n} x_{i}^{0}, \\
& \lambda_{i}=\left(X_{i}-2 x_{i}^{0}\right) G_{i}\left(x_{i}^{0}\right)\left(F\left(S^{0}\right)-F_{i}^{T}\right), \quad i=1, \ldots, n .
\end{aligned}
$$

We observe that the $n+1$ eigenvalues of $J^{0}$ are real, $\lambda_{0}$ being always negative. However, for $i=1, \ldots, n$, the sign of $\lambda_{i}$ depends on the values of $\left(X_{i}-2 x_{i}^{0}\right)$ and $\left(F\left(S^{0}\right)-F_{i}^{T}\right)$. More precisely, $\lambda_{i}$ is negative if and only if $x_{i}^{0}=0$ and $S^{0}<S_{i}^{T}$, or, $x_{i}^{0}=X_{i}$ and $S^{0}>S_{i}^{T}$. Thus $\lambda_{i}<0$ if and only if $i \in P_{1}$ or $i \in P_{2}$.

For any critical point in $E_{2}$, we have:

$$
\begin{aligned}
J_{0 j} & =-W_{r}^{T}, \quad j=1, \ldots, n, \\
J_{i 0} & =0, \quad i=1, \ldots, n, \quad i \neq r, \\
J_{r 0} & =x_{r}^{0}\left(X_{r}-x_{r}^{0}\right) G_{r}\left(x_{r}^{0}\right) \frac{\mathrm{d} F}{\mathrm{~d} S}\left(S_{r}^{T}\right), \\
J_{i i} & =\left(X_{i}-2 x_{i}^{0}\right) G_{i}\left(x_{i}^{0}\right)\left(F_{r}^{T}-F_{i}^{T}\right), \quad i=1, \ldots, n, i \neq r, \\
J_{r r} & =0 .
\end{aligned}
$$

Although $J^{0}$ is not triangular, its first column contains only two non-zero elements and a simple computation shows that its eigenvalues are:

$$
\begin{aligned}
& \lambda_{i}=\left(X_{i}-2 x_{i}^{0}\right) G_{i}\left(x_{i}^{0}\right)\left(F_{r}^{T}-F_{i}^{T}\right), \quad i=1, \ldots, n, i \neq r, \\
& \lambda_{0}=\frac{1}{2}\left(-A-\frac{\mathrm{d} W}{\mathrm{~d} S}\left(S_{r}^{T}\right) \sum_{i=1}^{n} x_{i}^{0}-\sqrt{\Delta}\right), \\
& \lambda_{r}=\frac{1}{2}\left(-A-\frac{\mathrm{d} W}{\mathrm{~d} S}\left(S_{r}^{T}\right) \sum_{i=1}^{n} x_{i}^{0}+\sqrt{\Delta}\right),
\end{aligned}
$$

where

$$
\Delta=\left(A+\frac{\mathrm{d} W}{\mathrm{~d} S}\left(S_{r}^{T}\right) \sum_{i=1}^{n} x_{i}^{0}\right)^{2}-4 x_{r}^{0}\left(X_{r}-x_{r}^{0}\right) G_{r}\left(x_{r}^{0}\right) \frac{\mathrm{d} F}{\mathrm{~d} S}\left(S_{r}^{T}\right) W_{r}^{T} .
$$


The real part of $\lambda_{0}$ is always negative and the same property holds for $\lambda_{r}$ if and only if $4 x_{r}^{0}\left(X_{r}-x_{r}^{0}\right) G_{r}\left(x_{r}^{0}\right) \frac{\mathrm{d} F}{\mathrm{~d} S}\left(S_{r}^{T}\right) W_{r}^{T}>0$, or equivalently $0<x_{r}^{0}<X_{r}$. Thus $\operatorname{Re}\left(\lambda_{r}\right)<0$ if and only if $r \in P_{3}$.

For $i \neq 0$ and $i \neq r$, we see that $\lambda_{i}$ is real, its sign depending on the values of $\left(X_{i}-2 x_{i}^{0}\right)$ and $\left(F_{r}^{T}-F_{i}^{T}\right)$. More precisely, $\lambda_{i}$ is negative if and only if $x_{i}^{0}=0$ and $S_{r}^{T}<S_{i}^{T}$, or, $x_{i}^{0}=X_{i}$ and $S_{r}^{T^{r}}>S_{i}^{T}$. Thus $\lambda_{i}<0$ if and only if $i \in P_{1}$ or $i \in P_{2}$.

Consequently system (1) admits one and only one locally stable critical point in the physiological domain.

\subsection{Global stability}

Theorem 3. The solutions of system (1) with initial conditions in $D^{\circ}$ have the following properties:

(i) if $0 \leqslant Q \leqslant A S_{1}^{T}$, then

$$
\begin{aligned}
& \lim _{t \rightarrow \infty} S(t)=S_{1}^{*}=\frac{Q}{A}, \\
& \lim _{t \rightarrow \infty} x_{i}(t)=0 \quad \text { for } 1 \leqslant i \leqslant n,
\end{aligned}
$$

(ii) if $A S_{1}^{T}<Q<A S_{1}^{T}+W_{1}^{T} X_{1}$, then

$\lim _{t \rightarrow \infty} S(t)=S_{1}^{T}$,

$\lim _{t \rightarrow \infty} x_{1}(t)=x_{1}^{*}=\frac{Q-A S_{1}^{T}}{W_{1}^{T}}$,

$\lim _{t \rightarrow \infty} x_{i}(t)=0 \quad$ for $2 \leqslant i \leqslant n$,

(iii) if $A S_{r-1}^{T}+W_{r-1}^{T} \sum_{i=1}^{r-1} X_{i} \leqslant Q \leqslant A S_{r}^{T}+W_{r}^{T} \sum_{i=1}^{r-1} X_{i}$ for $2 \leqslant r \leqslant n$, then

$\lim _{t \rightarrow \infty} S(t)=S_{r}^{*} \quad$ where $W\left(S_{r}^{*}\right)=\frac{Q-A S_{r}^{*}}{\sum_{i=1}^{r-1} X_{i}}$,

$\lim _{t \rightarrow \infty} x_{i}(t)=X_{i} \quad$ for $1 \leqslant i \leqslant r-1$,

$\lim _{t \rightarrow \infty} x_{i}(t)=0 \quad$ for $r \leqslant i \leqslant n$,

(iv) if $A S_{r}^{T}+W_{r}^{T} \sum_{i=1}^{r-1} X_{i}<Q<A S_{r}^{T}+W_{r}^{T} \sum_{i=1}^{r} X_{i}$ for $2 \leqslant r \leqslant n$, then

$\lim _{t \rightarrow \infty} S(t)=S_{r}^{T}$,

$\lim _{t \rightarrow \infty} x_{i}(t)=X_{i} \quad$ for $1 \leqslant i \leqslant r-1$,

$\lim _{t \rightarrow \infty} x_{r}(t)=x_{r}^{*}=\frac{Q-A S_{r}^{T}}{W_{r}^{T}}-\sum_{i=1}^{r-1} X_{i}$,

$\lim _{t \rightarrow \infty} x_{i}(t)=0 \quad$ for $r+1 \leqslant i \leqslant n$, 
(v) if $A S_{n}^{T}+W_{n}^{T} \sum_{i=1}^{n} X_{i} \leqslant Q<\infty$, then

$\lim _{t \rightarrow \infty} S(t)=S_{n+1}^{*} \quad$ where $W\left(S_{n+1}^{*}\right)=\frac{Q-A S_{n+1}^{*}}{\sum_{i=1}^{n} X_{i}}$

$\lim _{t \rightarrow \infty} x_{i}(t)=X_{i} \quad$ for $1 \leqslant i \leqslant n$.

Although we shall proceed as in $[47,66]$ by introducing a Lyapunov function and by using LaSalle's theorem [64,65], technical points have to be verified in this new situation. In certain respects, all situations are treated in a similar way and we start the discussion with (iii).

Proof of (iii). We first introduce the following open set $U_{\Delta} \subset \mathbb{R}^{n+1}$ :

$$
\begin{aligned}
U_{\Delta} & =\left\{\left(S, x_{1}, \ldots, x_{n}\right) \mid S>0, \quad 0<x_{i}<X_{i}+\Delta, \quad i=1, \ldots, r-1,-\Delta<x_{i}<X_{i},\right. \\
i & =r, \ldots, n\}, \Delta>0 .
\end{aligned}
$$

According to our basic assumptions, the right-hand member of system (1) is well defined and continuously differentiable over $U_{\Delta}$. Let us also introduce the function:

$$
V\left(S, x_{1}, \ldots, x_{n}\right)=\int_{S_{r}^{*}}^{S} \frac{F(s)-F\left(S_{r}^{*}\right)}{W(s)} \mathrm{d} s-\sum_{i=1}^{r-1} \int_{X_{i}}^{x_{i}} \frac{\mathrm{d} x}{x G_{i}(x)}+\sum_{i=r}^{n} \int_{0}^{x_{i}} \frac{\mathrm{d} x}{\left(X_{i}-x\right) G_{i}(x)},
$$
where $S_{r}^{*}$ is determined by $W\left(S_{r}^{*}\right)=\frac{Q-A S_{r}^{*}}{\sum_{i=1}^{r-1} X_{i}}$. Since $A S_{r-1}^{T}+W_{r-1}^{T} \sum_{i=1}^{r-1} X_{i} \leqslant Q \leqslant A S_{r}^{T}+W_{r}^{T} \sum_{i=1}^{r-1} X_{i}$
hold, we have $S_{r-1}^{T} \leqslant S_{r}^{*} \leqslant S_{r}^{T}$.

$V$ is clearly well defined and continuously differentiable over $U_{\Delta}$ and we show that it is a Lyapunov function for system (1) over $U_{\Delta}$. For every $c>0$, the subset $\Omega_{c}$ of $U_{\Delta}$ defined by

$$
\begin{aligned}
\Omega_{c} & =\left\{\left(S, x_{1}, \ldots, x_{n}\right) \in U_{\Delta} \mid S>0, \quad 0<x_{i} \leqslant X_{i}, i=1, \ldots, r-1,0 \leqslant x_{i}<X_{i},\right. \\
i & \left.=r, \ldots, n \text { and } V\left(S, x_{1}, \ldots, x_{n}\right) \leqslant c\right\} .
\end{aligned}
$$

First, we show that $\Omega_{c}$ is a compact subset of $\mathbb{R}^{\mathrm{m}+1}$. On the one hand, $\Omega_{c}$ is bounded since for points $\left(S, x_{1}, \ldots, x_{n}\right) \in U_{\Delta}, V \rightarrow \infty$ if $S \rightarrow \infty$. On the other hand, for points in $U_{\Delta}, V \rightarrow \infty$ if $S \rightarrow 0$ or $x_{i} \rightarrow 0$ (for $1 \leqslant i \leqslant r-1$ ) or $x_{i} \rightarrow X_{i}$ (for $r \leqslant i \leqslant n$ ). Thus every limit point $\left(\tilde{S}, \tilde{x}_{1}, \ldots, \tilde{x}_{n}\right)$ of $\Omega_{c}$ satisfies $\tilde{S}>0, \tilde{x}_{i}>0$ (for $\left.1 \leqslant i \leqslant r-1\right)$ and $\tilde{x}_{i}>X_{i}$ (for $r \leqslant i \leqslant n$ ). The continuity of $V$ entails that every limit point of $\Omega_{c}$ belongs to the latter proving that this set is also closed and hence compact.

A straightforward computation provides:

$$
\begin{aligned}
\dot{V}\left(S, x_{1}, \ldots, x_{n}\right)= & \frac{F(S)-F\left(S_{r}^{*}\right)}{W(S)}\left(Q-A S-W(S) \sum_{i=1}^{r-1} X_{i}\right)-\sum_{i=1}^{r-1}\left(X_{i}-x_{i}\right)\left(F\left(S_{r}^{*}\right)-F_{i}^{T}\right) \\
& +\sum_{i=r}^{n} x_{i}\left(F\left(S_{r}^{*}\right)-F_{i}^{T}\right),
\end{aligned}
$$

and we now discuss the sign of $\dot{V}$ over $\Omega_{c}$. Consequently of $S_{r-1}^{T} \leqslant S_{r}^{*} \leqslant S_{r}^{T}$ together with the strict monotonicity of $F$, the second and the third terms in the right-hand member of the preceding equation are non-positive over $\Omega_{c}$. Moreover, the definition of $S_{r}^{*}$ shows that it is a zero 
$H(S)=Q-A S-W(S) \sum_{i=1}^{r-1} X_{i}$. Since $\frac{\mathrm{d} H}{\mathrm{~d} S}=-A-\frac{\mathrm{d} W}{\mathrm{~d} S} \sum_{i=1}^{r-1} X_{i}<0, H(S)$ is positive over $\left[0, S_{r}^{*}\right)$ and negative over $\left(S_{r}^{*}, \infty\right)$. Consequently, $\frac{F(S)-F\left(S_{r}^{*}\right)}{W(S)} H(S)$ is non-positive over $(0, \infty)$ and thus $\dot{V} \leqslant 0$ over $\Omega_{c}$. We conclude that $\Omega_{c}$ is invariant for our differential system and that $V$ is a Lyapunov function over the preceding set. It is important to note that, because $V$ goes to $+\infty$ as its argument tends to the boundary of $D$, every path starting in $D^{\circ}$ can be imbedded into some $\Omega_{c}$ for a suitable choice of c. According to LaSalle's theorem [64,65], every such solution converges to the largest invariant subset $M_{\Omega_{c}}$ of $E_{\Omega_{c}}=\left\{y \in \Omega_{c} ; \dot{V}(y)=0\right\}$.

We distinguish three subcases:

(1) If $A S_{r-1}^{T}+W_{r-1}^{T} \sum_{i=1}^{r-1} X_{i}<Q<A S_{r}^{T}+W_{r}^{T} \sum_{i=1}^{r-1} X_{i}$, then $S_{r-1}^{T}<S_{r}^{*}<S_{r}^{T}$ and the preceding considerations about $\dot{V}$ entail:

$M_{\Omega_{c}}=E_{\Omega_{c}}=\left\{\left(S_{r}^{*}, X_{1}, \ldots, X_{r-1}, 0, \ldots, 0\right)\right\}$,

(2) If $Q=A S_{r-1}^{T}+W_{r-1}^{T} \sum_{i=1}^{r-1} X_{i}$, then $S_{r}^{*}=S_{r-1}^{T}$ and for the same reasons as above we get:

$E_{\Omega_{c}}=\left\{\left(S_{r}^{*}, X_{1}, \ldots, X_{r-2}, x_{r-1}, 0, \ldots, 0\right) \mid x_{r-1} \in\left(0, X_{r-1}\right]\right\}$.

We consider a solution of system (1) with initial condition in the largest invariant subset $M_{\Omega_{c}}$ of $E_{\Omega_{c}}$. Clearly, $\dot{x}_{i}(0)=0$ for $i=1, \ldots, r-2, r, \ldots, n$ and, as a consequence of invariance, $\dot{S}(0)=0$. Since $S(0)=S_{r-1}^{T}, \dot{x}_{r-1}(0)=0$ and the starting point is thus a fixed point. Moreover, $Q=A S_{r-1}^{T}+W_{r-1}^{T} \sum_{i=1}^{r-1} X_{i}$ and therefore $\dot{S}(0)=W_{r-1}^{T}\left(X_{r-1}-x_{r-1}(0)\right)$ entailing $x_{r-1}(0)=$ $X_{r-1}$. We conclude:

$$
M_{\Omega_{c}}=\left\{\left(S_{r}^{*}, X_{1}, \ldots, X_{r-1}, 0, \ldots, 0\right)\right\} .
$$

(3) If $Q=A S_{r}^{T}+W_{r}^{T} \sum_{i=1}^{r-1} X_{i}$, then $S_{r}^{*}=S_{r}^{T}$ and a reasoning similar to the preceding one provides $E_{\Omega_{c}}=\left\{\left(S_{r}^{*}, X_{1}, \ldots, X_{r-1}, x_{r}, 0, \ldots, 0\right) \mid x_{r} \in\left[0, X_{r}\right)\right\} \quad$ and $\quad$ since $\dot{S}(0)=-W_{r}^{T} x_{r}(0)=0$, we conclude:

$$
M_{\Omega_{c}}=\left\{\left(S_{r}^{*}, X_{1}, \ldots, X_{r-1}, 0, \ldots, 0\right)\right\} .
$$

All three configurations (1)-(3) lead to $M_{\Omega_{c}}=\left\{\left(S_{r}^{*}, X_{1}, \ldots, X_{r-1}, 0, \ldots, 0\right)\right\}$ and thus, according to LaSalle's theorem $[64,65]$, we have:

$$
S(t) \stackrel{t \rightarrow \infty}{\rightarrow} S_{r}^{*}, \quad x_{i}(t) \stackrel{t \rightarrow \infty}{\rightarrow} X_{i}, \quad 1 \leqslant i \leqslant r-1 \text { and } x_{i}(t) \stackrel{t \rightarrow \infty}{\rightarrow} 0, \quad r \leqslant i \leqslant n,
$$

for every solution of system (1) with initial condition in the interior of $D$.

For the proof of the other points (i), (ii), (iv) and (v), we replace respectively the preceding Lyapunov function by:

(i) $V\left(S, x_{1}, \ldots, x_{n}\right)=\int_{S_{1}^{*}}^{S} \frac{F(s)-F\left(S_{1}^{*}\right)}{W(s)} \mathrm{d} s+\sum_{i=1}^{n} \int_{0}^{x_{i}} \frac{\mathrm{d} x}{\left(X_{i}-x\right) G_{i}(x)}$,

(ii) $V\left(S, x_{1}, \ldots, x_{n}\right)=\int_{S_{1}^{T}}^{S} \frac{F(s)-F_{1}^{T}}{W(s)} \mathrm{d} s+\int_{x_{1}^{*}}^{x_{1}} \frac{\left(x-x_{1}^{*}\right) \mathrm{d} x}{x\left(X_{1}-x\right) G_{1}(x)}+\sum_{i=2}^{n} \int_{0}^{x_{i}} \frac{\mathrm{d} x}{\left(X_{i}-x\right) G_{i}(x)}$,

(iv) $V\left(S, x_{1}, \ldots, x_{n}\right)=\int_{S_{r}^{T}}^{S} \frac{F(s)-F\left(S_{r}^{T}\right)}{W(s)} \mathrm{d} s \sum_{i=1}^{r-1} \int_{X_{i}}^{x_{i}} \frac{\mathrm{d} x}{x G_{i}(x)}+\int_{x_{r}^{*}}^{x_{r}} \frac{\left(x-x_{r}^{*}\right) \mathrm{d} x}{x\left(X_{r}-x\right) G_{r}(x)}+\sum_{i=r+1}^{n} \int_{0}^{x_{i}} \frac{\mathrm{d} x}{\left(X_{i}-x\right) G_{i}(x)}$,

(v) $V\left(S, x_{1}, \ldots, x_{n}\right)=\int_{S_{n+1}^{*}}^{S} \frac{F(s)-F\left(S_{n+1}^{*}\right)}{W(s)} \mathrm{d} s-\sum_{i=1}^{n} \int_{X_{i}}^{x_{i}} \frac{\mathrm{d} x}{x G_{i}(x)}$

and repeat the same argumentation. 


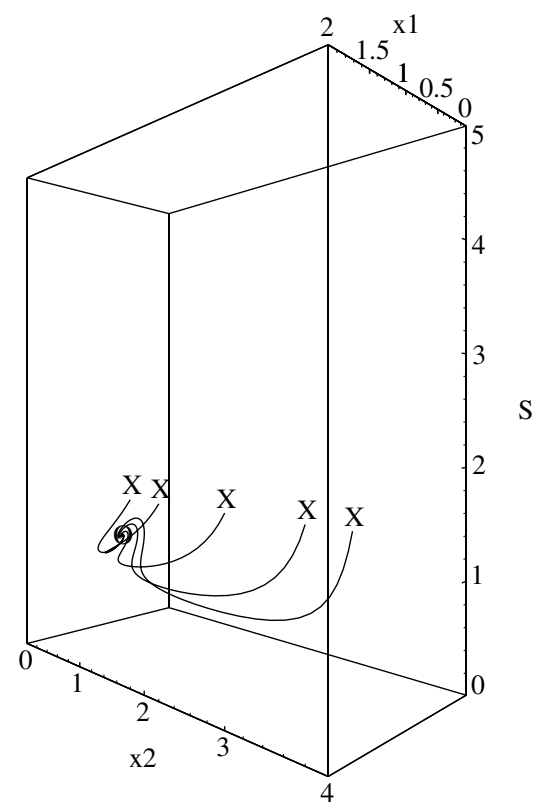

Fig. 1. Solutions of system (1) $W(S)=F(S)=B S /(K+S)$, for five different initial conditions, namely $(1.5,1,0.5)$, $(1.5,1,1),(1.5,1,2),(1.5,1,3),(1.5,1,3.5) ; A=0.25, B=10^{-11}, K=2, G_{i}(x)=2, i=1,2, X_{1}=2, X_{2}=4, S_{1}^{T}=1, S_{2}^{T}=2$, $Q=0.5$ with the units $[t]=10 \mathrm{~h},[S]=10^{-11} \mathrm{M},\left[x_{i}\right]=\left[X_{i}\right]=1,[K]=10^{-11} \mathrm{M},[Q]=\frac{10^{-11} \mathrm{M}}{10 \mathrm{~h}},[A]=\frac{1}{10 \mathrm{~h}},[B]=M$.

Obviously, the case $S_{1}^{T}=S_{2}^{T}=\cdots=S_{n}^{T}$ is singular in the parameter space and thus not basically relevant from a physiological viewpoint. We would nevertheless like to point out that convergence of the solutions of our differential system can be established by using the Frobenius integrability theorem and that the argument can be found in [48]. We do not discuss it here since it requires a very different approach.

The simulation (Fig. 1) illustrates the behaviour of the system with biologically reasonable parameters.

\section{Discussion}

\subsection{Limitations of the model}

A significant simplification in our model is that we have neglected most biological effects of the low affinity neurotrophin receptor, taking account only of its role in depleting extracellular NGF by endocytosis. The roles of this receptor and its relationship to the p75NTR protein are multiple and complex, and currently controversial [39]. When functioning as low affinity receptor, its binding of NGF or other neurotrophins causes their endocytosis and retrograde transport, and the initiation of several different signaling pathways that can promote both neuronal survival and neuronal death [38]. In addition, p75NTR can interact with trkA to enhance its activation by 
NGF [67] and acting on its own can bind pro-NGF (but not NGF) with high affinity [39]. While the existence of these effects has been clearly demonstrated in several parts of the nervous system, and while the genetic deletion of p75NTR has been shown to affect the outgrowth of sensory axons [68], and to lead to a loss of $50 \%$ of the neurons of dorsal root ganglia [69], current knowledge is not yet sufficient for these interactions to be modeled mathematically. For example, it is not clear whether the effects of p75NTR deletion on sensory neurons result from the absence of p75NTR in those neurons, or in Schwann cells, whose migration is likewise affected [68]. However, this simplification in our model may not be a serious defect, because the levels of NGF in the skin at the times of interaxonal competition are believed to be in the concentration range of the high affinity receptor $\left(K_{d \mathrm{~L}} \approx 10^{-11} \mathrm{M}\right)$, too low to affect the low affinity receptor $\left(K_{d \mathrm{~L}} \approx 10^{-9} \mathrm{M}\right)$. This remains uncertain, however, because the published measurements of NGF concentration, 40-80 pg/mg protein in whisker pads of mouse embryos [23], corresponding to about (3-6) $10^{-12} \mathrm{M}$, refer to average values, and the concentrations in the extracellular space available to axon terminals are unknown.

Another simplification is that we have assumed each axon, or axon branch, to be an independent entity. It is unknown whether this is true, but several computational studies have shown that interaction (by intracellular signals?) between an axon's branches could seriously affect the outcome $[49,70]$.

\subsection{Implications of this study}

Most previous attempts at modeling interaxonal competition were intended to explain the loss of polyneuronal innervation at sites such as the neuromuscular junction $[49,70,71]$. The winner-takes-all behavior of such models, or 'competitive exclusion', well reflects certain situations, but, as mentioned in the Introduction, other situations exist where multiple innervation of a small region can persist, and it turns out to be quite difficult to devise models of this. The present one is a modification of one of the competitive exclusion models [47], but obtains the persistence of multiple innervation by setting a limit to the growth of each axon. We extend and generalize the earlier mathematical analysis in which the fittest axon became so strong that it eliminated all the others. Our model predicts that with low levels of NGF production, only a single axon will persist in a touch dome (as in the former model), but that with higher levels several will persist, as appears to be the case in transgenic mice overexpressing NGF in the skin [46].

Other recent models of interaxonal competition have obtained the persistence of multiple innervation in several ways. This can be achieved by postulating that the axons depend on more than one trophic factor or are spatially separated [72]. In a special case of one of the competitive exclusion models, several axons can coexist if the concentration of trophic factor for zero growth is the same in each but lower than for all the other axons [47]. In other models, axonal coexistence results, as in the present paper, from their reaching a maximal growth limit. Thus, two models of Van Ooyen and Willshaw [73,74], imply an upper bound on the number of receptors per axon and on the rate at which trophic factor receptors can be inserted. A maximal growth limit is likewise inherent in some computer simulations of Elliott and Shadbolt (see Fig. 2 of Ref. [75]). 


\section{Acknowledgments}

We are grateful to Dr. Kathryn Albers and Dr. Arjen van Ooyen for comments on the manuscript, and to the Swiss National Science Foundation for support (grant 31.37409.93).

\section{References}

[1] P.G.H. Clarke, Neuronal death in the development of the vertebrate nervous system, Semin. Neurosci. 6 (1994) 291.

[2] V. Hamburger, R. Levi-Montalcini, Proliferation, differentiation and degeneration in the spinal ganglia of the chick embryo under normal and experimental conditions, J. Exp. Zool. 111 (1949) 457.

[3] M. Catsicas, Y. Péquignot, P.G.H. Clarke, Rapid onset of neuronal death induced by blockade of either axoplasmic transport or action potentials in afferent fibers during brain development, J. Neurosci. 12 (1992) 4642.

[4] L. Galli-Resta, M. Ensini, E. Fusco, A. Gravina, B. Margheritti, Afferent spontaneous electrical activity promotes the survival of target cells in the developing retinotectal system of the rat, J. Neurosci. 13 (1993) 243.

[5] M.-P. Primi, P.G.H. Clarke, Presynaptic initiation by action potentials of retrograde signals in developing neurons, J. Neurosci. 17 (1997) 4253.

[6] R. Levi-Montalcini, The nerve growth factor 35 years later, Science 237 (1987) 1154.

[7] H.F. Figueiredo, B.M. Davis, K.M. Albers, Skin-derived nerve growth factor blocks programmed cell death in the trigeminal ganglia but does not enhance neuron proliferation, Mech. Devel. 109 (2001) 205.

[8] R.S. Goldstein, Axial level-dependent differences in size of avian dorsal root ganglia are present from gangliogenesis, J. Neurobiol. 24 (1993) 1121.

[9] V.M. Carr, S.B. Simpson Jr., Proliferative and degenerative events in the early development of chick dorsal root ganglia. I. Normal development, J. Comp. Neurol. 182 (1978) 727.

[10] S.R. Detwiler, On the hyperplasia of nerve centers resulting from excessive peripheral loading, Proc. Nat. Acad. Sci. USA 6 (2003) 96.

[11] E.D. Bueker, Hyperplastic changes in the nervous system of a frog (Rana) as associated with multiple functional limbs, Anat. Rec. 93 (2003) 323.

[12] M. Hollyday, V. Hamburger, Reduction of the naturally occurring motor neuron loss by enlargement of the periphery, J. Comp. Neurol. 170 (1976) 311.

[13] D.L. Kitson, A. Roberts, Competition during innervation of embryonic amphibian head skin, Proc. Roy. Soc. Lond. B 218 (1983) 49.

[14] S.A. Scott, The effects of neural crest deletions on the development of sensory innervation patterns in embryonic chick hind limb, J. Physiol. 352 (1984) 285.

[15] P. Ernfors, K.-F. Lee, J. Kucera, R. Jaenisch, Lack of neurotrophin-3 leads to deficiencies in the peripheral nervous system and loss of limb proprioceptive afferents, Cell 77 (1994) 503.

[16] I. Fariñas, K.R. Jones, C. Backus, X.-Y. Wang, L.F. Reichardt, Severe sensory and sympathetic deficits in mice lacking neurotrophin-3, Nature 369 (1994) 658.

[17] F. Hory-Lee, M. Russell, R.M. Lindsay, E. Frank, Neurotrophin 3 supports the survival of developing muscle sensory neurons in culture, Proc. Nat. Acad. Sci. USA 90 (1993) 2613.

[18] A.M. LeMaster, R.F. Krimm, B.M. Davis, T. Noel, M.E. Forbes, J.E. Johnson, K.M. Albers, Overexpression of brain-derived neurotrophic factor enhances sensory innervation and selectively increases neuron number, J. Neurosci. 19 (1999) 5919.

[19] K.M. Albers, T.N. Perrone, T.P. Goodness, M.E. Jones, M.A. Green, B.M. Davis, Cutaneous overexpression of NT-3 increases sensory and sympathetic neuron number and enhances touch dome and hair follicle innervation, J. Cell Biol. 134 (1996) 487.

[20] R. Klein, I. Silos-Santiago, R.J. Smeyne, S.A. Lira, R. Brambilla, S. Bryant, L. Zhang, W.D. Snider, M. Barbacid, Disruption of the neurotrophin-3 receptor gene trkC eliminates Ia muscle afferents and results in abnormal movements, Nature 368 (1994) 249. 
[21] M. Zwick, B.M. Davis, C.J. Woodbury, J.N. Burkett, H.R. Koerber, J.F. Simpson, K.M. Albers, Glial cell linederived neurotrophic factor is a survival factor for isolectin B4-positive, but not vanilloid receptor 1-positive, neurons in the mouse, J. Neurosci. 22 (2002) 4057.

[22] R. Heumann, S. Korsching, J. Scott, H. Thoenen, Relationship between levels of nerve growth factor (NGF) and its messenger RNA in sympathetic ganglia and peripheral target tissues, EMBO J. 3 (1984) 3183.

[23] A.M. Davies, C. Bandtlow, R. Heumann, S. Korsching, H. Rohrer, H. Thoenen, Timing and site of nerve growth factor synthesis in developing skin in relation to innervation and expression of the receptor, Nature 326 (1987) 353.

[24] S. Korsching, H. Thoenen, Treatment with 6-hydroxydopamine and colchicine decreases nerve growth factor levels in sympathetic ganglia and increases them in the corresponding target tissues, J. Neurosci. 5 (1985) 1058.

[25] K.M. Albers, D.E. Wright, B.M. Davis, Overexpression of nerve growth factor in epidermis of transgenic mice causes hypertrophy of the peripheral nervous system, J. Neurosci. 14 (1994) 1422.

[26] S.M. Harrison, B.M. Davis, M. Nishimura, K.M. Albers, M.E. Jones, H.S. Phillips, Rescue of NGF-deficient mice I: transgenic expression of NGF in skin rescues mice lacking endogenous NGF, Molec. Brain Res. 122 (2) (2004) 116.

[27] R. Inbal, M. Rousso, H. Ashur, P.D. Wall, M. Devor, Collateral sprouting in skin and sensory recovery after nerve injury in man, Pain 28 (1987) 141.

[28] J. Diamond, M. Holmes, M. Coughlin, Endogenous NGF and nerve impulses regulate the collateral sprouting of sensory axons in the skin of the adult rat, J. Neurosci. 12 (1992) 1454.

[29] K.B. English, S. Harper, N. Stayner, Z.-M. Wang, A.M. Davies, Localization of nerve growth factor (NGF) and low-affinity NGF receptors in touch domes and quantification of NGF mRNA in keratinocytes of adult rats, J. Comp. Neurol. 344 (1994) 470.

[30] B. Doubleday, P.P. Robinson, Nerve growth factor depletion reduces collateral sprouting of cutaneous mechanoreceptive and tooth-pulp axons in ferrets, J. Physiol. 481 (1994) 709.

[31] A.H. Lamb, Motoneurone counts in Xenopus frogs reared with one bilaterally-innervated hindlimb, Nature 284 (1980) 347.

[32] A.H. Lamb, M.J. Ferns, K. Klose, Peripheral competition in the control of sensory neuron numbers in Xenopus frogs reared with a single bilaterally innervated hindlimb, Dev. Brain Res. 45 (1989) 149.

[33] H. Thoenen, Neurotrophins and activity-dependent plasticity, Prog. Brain Res. 128 (2000) 183.

[34] H. Rohrer, R. Heumann, H. Thoenen, The synthesis of nerve growth factor (NGF) in developing skin is independent of innervation, Dev. Biol. 128 (1988) 240.

[35] I. Matsuoka, M. Meyer, H. Thoenen, Cell-type-specific regulation of nerve growth factor (NGF) synthesis in nonneuronal cells: comparison of Schwann cells with other cell types, J. Neurosci. 11 (1991) 3165.

[36] V.L. Buchman, A.M. Davies, Different neurotrophins are expressed and act in a developmental sequence to promote the survival of embryonic sensory neurons, Development 118 (1993) 989.

[37] C.F. Ibanez, P. Ernfors, T. Timmusk, N.Y. Ip, E. Arenas, G.D. Yancopoulos, H. Persson, Neurotrophin-4 is a target-derived neurotrophic factor for neurons of the trigeminal ganglion, Development 117 (1993) 1345.

[38] E.J. Huang, L.F. Reichardt, Neurotrophins: roles in neuronal development and function, Ann. Rev. Neurosci. 24 (2001) 677.

[39] M.V. Chao, M. Bothwell, Neurotrophins: to cleave or not to cleave, Neuron. 33 (2002) 9.

[40] M. Barbacid, Structural and functional properties of the TRK family of neurotrophin receptors, Ann. NY Acad. Sci. 766 (1995) 442.

[41] D.C. Molliver, D.E. Wright, M.L. Leitner, A.S. Parsadanian, K. Doster, D. Wen, Q. Yan, W.D. Snider, IB4binding DRG neurons switch from NGF to GDNF dependence in early postnatal life, Neuron. 19 (1997) 849.

[42] J.C. Conover, G.D. Yancopoulos, Neurotrophin regulation of the developing nervous system: analyses of knockout mice, Rev. Neurosci. 8 (1997) 13.

[43] M. Tazaki, S. Sakada, Merkel cell-neurite complexes in touch domes of hamster cheek pouch, Bull. Tokyo. Dent. Coll. 35 (1994) 95.

[44] A. Davies, A. Lumsden, Relation of target encounter and neuronal death to nerve growth factor responsiveness in the developing mouse trigeminal ganglion, J. Comp. Neurol. 223 (1984) 124.

[45] U. Vielkind, M.K. Sebzda, I.R. Gibson, M.H. Hardy, Dynamics of Merkel cell patterns in developing hair follicles in the dorsal skin of mice, demonstrated by a monoclonal antibody to mouse keratin 8, Acta Anat. 152 (1995) 93. 
[46] B.M. Davis, B.T. Fundin, K.M. Albers, T.P. Goodness, K.M. Cronk, F.L. Rice, Overexpression of nerve growth factor in skin causes preferential increases among innervation to specific sensory targets, J. Comp. Neurol. 387 (1997) 489.

[47] N. Jeanprêtre, P.G.H. Clarke, J.P. Gabriel, Competitive exclusion between axons dependent on a single trophic substance: A mathematical analysis, Math. Biosci. 135 (1996) 23.

[48] R. Kohli, Analyse mathématique de la compétition entre les axones des neurones des ganglions sensitifs pour l'innervation d'une région de la peau, Thèse No 1334, Université de Fribourg, 2001.

[49] M.R. Bennett, J. Robinson, Growth and elimination of nerve terminals at synaptic sites during polyneuronal innervation of muscle cells: a trophic hypothesis, Proc. Roy. Soc. Lond. B 235 (1989) 299.

[50] J.R. Sanes, J.W. Lichtman, Development of the vertebrate neuromuscular junction, Ann. Rev. Neurosci. 22 (1999) 389.

[51] E. Cooper, J. Diamond, A quantitative study of the mechanosensory innervation of the salmander skin, J. Physiol. 264 (1977) 695.

[52] A.A. Verveen, Fields of touch receptors in the frog skin, Exp. Neurol. 8 (1963) 482.

[53] R. Kohli, P.G.H. Clarke, Mathematical analysis of competition between sensory ganglion cells for nerve growth factor in the skin, Lect. Notes Comput. Sci. 1327 (1997) 133.

[54] P.C. Letourneau, Chemotactic response of nerve fiber elongation to nerve growth factor, Dev. Biol. 66 (1978) 183.

[55] R.W. Gundersen, J.N. Barrett, Neuronal chemotaxis: chick dorsal-root axons turn toward high concentrations of nerve growth factor, Science 206 (1979) 1079.

[56] J.M. Aletta, L.A. Greene, Growth cone configuration and advance: a time-lapse study using video-enhanced differential interference contrast microscopy, J. Neurosci. 8 (1988) 1425.

[57] G. Dechant, A. Rodríguez-Tébar, Y.-A. Barde, Neurotrophin receptors, Prog. Neurobiol. 42 (1994) 347.

[58] S.O. Meakin, E.M. Shooter, The nerve growth factor family of receptors Trends Neurosci., Trends Neurosci. 15 (1992) 323.

[59] D. Colquhoun, The link between drug binding and response: theories and observations, in: R.D. O'Brien (Ed.), The Receptor: A Comprehensive Treatise, vol. 1, General Principles and Procedures, Plenum, New York, 1979, p. 93.

[60] M.W. Weible, S.E. Bartlett, A.J. Reynolds, I.A. Hendry, Prolonged recycling of internalized neurotrophins in the nerve terminal, Cytometry 43 (2001) 182.

[61] L. Perko, Differential Equations and Dynamical Systems, Springer, New York, 1991.

[62] P. Hartman, Ordinary Differential Equations, Birkhäuser, Boston, 1982.

[63] H. Amann, Gewöhnliche Differentialgleichungen, Walter de Gruyter, Berlin, 1983.

[64] J.P. LaSalle, Some extensions of Liapunov's second method, IRE Trans. Circuit Theory 7 (1960) 520.

[65] J.P. LaSalle, An invariance principle in the theory of stability, in: J. Hale, J.P. LaSalle (Eds.), Differential Equations and Dynamical Systems, Academic Press, New York, 1968, p. 277.

[66] B. Hsu, Limiting behavior for competing species, SIAM J. Appl. Math. 3 (1978) 760.

[67] A. Horton, G. Laramee, S. Wyatt, A. Schih, J. Winslow, A.M Davies, NGF binding to p75 enhances the sensitivity of sensory and sympathetic neurons to NGF at different stages of development, Mole. Cell Nerosci. 10 (1997) 162.

[68] C.A. Bentley, K.F. Lee, p75 is important for axon growth and schwann cell migration during development, J. Neurosci. 20 (2000) 7706.

[69] I. Bergmann, J.V. Priestley, S.B. McMahon, E.B. Brocker, K.V. Toyka, M. Koltzenburg, Analysis of cutaneous sensory neurons in transgenic mice lacking the low affinity neurotrophin receptor p75, Eur. J. Neurosci. 9 (1997) 18.

[70] C.E. Rasmussen, D.J. Willshaw, Presynaptic and postsynaptic competition in models for the development of neuromuscular connections, Biol. Cybern. 68 (1993) 409.

[71] J.L. Gouzé, J.M. Lasry, J.P. Changeux, Selective stabilization of muscle innervation during development: a mathematical model, Biol. Cybern. 46 (1983) 207.

[72] A. Van Ooyen, D.J. Willshaw, Development of nerve connections under the control of neurotrophic factors: parallels with consumer-resource systems in population biology, J. Theor. Biol. 206 (2000) 195. 
[73] A. Van Ooyen, D.J. Willshaw, Poly- and mononeuronal innervation in a model for the development of neuromuscular connections, J. Theor. Biol. 196 (1999) 495.

[74] A. Van Ooyen, D.J. Willshaw, Competition for neurotrophic factor in the development of nerve connections, Proc. Roy. Soc. Lond. B 266 (1999) 883.

[75] T. Elliott, N.R. Shadbolt, A mathematical model of activity-dependent, anatomical segregation induced by competition for neurotrophic support, Biol. Cybern. 75 (1996) 463. 